\title{
Aportaciones de las pedagogías de género a la calidad de la docencia universitaria
}

\author{
Maria Mena Yuste \\ Ángela Sáez Díaz \\ Aurora Leal \\ Margot Pujal Llombart \\ Universitat Autònoma de Barcelona. España. \\ mariamenayuste@gmail.com \\ saezdiazangela@gmail.com \\ aurora.leal@uab.cat \\ margot.pujal@uab.cat
}

Recibido: 22/3/2017

Aceptado: $13 / 3 / 2018$

Publicado: 15/7/2019

\section{Resumen}

A partir de la necesidad de introducir la perspectiva de género en la docencia debido al vacío existente en la mayoría de estudios de grado en Cataluña (España), sobre todo en Ciencias de la Salud y Psicología, se diseñó una oferta formativa de talleres. En ella se consideró básico introducir la perspectiva de género, tanto a nivel de contenido como metodológico. En este artículo se explora el relevante papel que la introducción de metodologías y pedagogías feministas puede tener en el aumento de la calidad de la docencia a partir de dicha experiencia a través de narrativas colectivas de personas formadoras y participantes. Estas narrativas fueron construidas a partir de cinco grupos de discusión, con un total de 20 personas en la muestra que fueron seleccionadas a partir de criterios de variabilidad y representatividad. Las dos narrativas visibilizan los procesos que se vivieron en los talleres y las transformaciones que plantea la integración de metodologías y pedagogías feministas en la construcción compartida del conocimiento. La principal conclusión que se extrae de ello es que la introducción de metodologías y epistemologías feministas permite reflexionar críticamente sobre nuestras prácticas como formadoras y estudiantes en la reproducción de las relaciones de poder de género en y fuera del aula para devenir sujetos activos de cambio.

Palabras clave: docencia con perspectiva de género; innovación pedagógica; calidad; educación superior; metodologías; narrativas

\section{Resum. Aportacions de les pedagogies de gènere a la qualitat de la docència universitària}

A partir de la necessitat d'introduir la perspectiva de gènere en la docència atès el buit existent a la majoria d'estudis de grau a Catalunya, sobretot a Ciències de la Salut i Psicologia, es va dissenyar una oferta formativa de tallers, en la qual es va considerar bàsic introduir la perspectiva de gènere, tant a nivell de contingut com metodològic. En aquest article s'hi explora el paper rellevant que la introducció de metodologies i pedagogies feministes pot tenir en l'augment de la qualitat de la docència a partir d'aquesta experiència amb narratives col.lectives de persones formadores i participants. Aquestes narratives van ser construïdes a partir 
de cinc grups de discussió, amb un total de 20 persones a la mostra que van ser seleccionades a partir de criteris de variabilitat i representativitat. Les dues narratives visibilitzen els processos que es van viure als tallers i també les transformacions que planteja la integració de metodologies i pedagogies feministes en la construcció compartida del coneixement. La conclusió principal que se n'extreu és que la introducció de metodologies i epistemologies feministes permet reflexionar críticament sobre les nostres pràctiques com a formadores i estudiants en la reproducció de les relacions de poder de gènere dins i fora de l'aula per esdevenir subjectes actius de canvi.

Paraules clau: docència amb perspectiva de gènere; innovació pedagògica; qualitat, educació superior; metodologies; narratives

\section{Abstract. Contributions of gender pedagogies to teaching quality in higher education}

Most undergraduate programs of study in Catalonia, Spain, especially in the health sciences and psychology, lack a gender perspective in teaching. To fill this gap, a series of training workshops were designed with a gender perspective in content and methodology. This article explores the important role that the introduction of feminist methodologies and pedagogies can have in enhancing the quality of teaching through an experience using collective narratives of teachers and participants. The narratives were constructed from five discussion groups with a total sample of 20 people selected according to criteria of variability and representativeness. The two narratives reveal the processes the participants experienced during the workshops and how the integration of feminist methodologies and pedagogical approaches transform the shared construction of knowledge. The main conclusion is that the introduction of feminist methodologies and epistemologies allow us to critically reflect on our practices as trainers and students in the reproduction of gender power relations in and outside the classroom to become active subjects of change.

Keywords: gender perspective in teaching; pedagogical innovation; quality; higher education; methodologies; narratives

\begin{aligned} & \multicolumn{2}{c}{ Sumario } \\ & 1. Introducción 4. Método \\ & 2. Las pedagogías de género 5. Resultados \\ & en la calidad de la docencia 6. Discusión y conclusiones \\ & 3. Objetivo Referencias bibliográficas \end{aligned}

\section{Introducción}

La calidad de la educación superior es un tema que viene preocupando en los ámbitos académicos. Sin embargo, acerca de lo que se considera calidad puede darse una ruptura epistemológica entre dos tipos de concepciones: aquella que es vista como la respuesta adecuada y la que es considerada una pregunta, un propósito a desarrollar.

La transformación que tiene - y debe tener — lugar en la persona estudiante de educación universitaria requiere, además de dominar una cantidad crítica de datos y teorías, saber hacerse con una gama de conocimientos múltiples de 
acción, de autoidentidad y de riesgo, aspectos no siempre bien comprendidos desde los referentes académicos (Rué, 2015). Se trata de empoderar y mejorar la inclusión de las participantes y los participantes en la relación educativa. Los resultados no pueden separarse de los procesos, por lo que estos deben también ser objeto de atención y de análisis. Es importante también introducir el elemento subjetivo en los análisis de situaciones sometidas a investigación (Harding, 1987). Este planteamiento puede definirse como rasgo metodológico, pero también puede concebirse como característica epistemológica, porque implica teorías del conocimiento diferentes de las tradicionales. El conocimiento, tal como lo expresa Haraway (1995), debería difractar la realidad, más que reflejarla, para que esta no quedara atrapada en una única perspectiva.

Consideramos relevante producir conocimiento científico sobre género y educación, ya que ello se traducirá en cambios en las prácticas educativas, en las percepciones del mundo y en las relaciones con los demás (Colás, 2003). Un planteamiento metodológico feminista favorece la introducción de una concepción distinta y complementaria de lo que se suele entender como calidad de la educación superior (Donoso-Vázquez y Velasco Martínez, 2013; Grünberg, 2011).

\section{Las pedagogías de género en la calidad de la docencia}

Las primeras reivindicaciones feministas a nivel universitario iban orientadas al acceso de las mujeres al conocimiento científico. Posteriormente, los esfuerzos se dirigieron a la coeducación, entendida primero como un trato no discriminatorio e igualitario en las aulas y en la investigación, y más adelante como la revisión de los modelos organizativos y curriculares para que incorporasen la experiencia, las aptitudes y las aportaciones sociales y culturales de las mujeres y los hombres sin estereotipos sexistas ni androcéntricos ni tampoco actitudes discriminatorias (Luxán y Biglia, 2011). En este artículo se propone ir un poco más allá de la coeducación y dirigirse hacia una pedagogía de género que proponga teoría, modelos y estrategias reales para la incorporación del género en el currículo y en el aula (Colás, 2004). La introducción de la perspectiva de género en la docencia supone someter los diferentes aspectos que la componen (contenido, metodologías, interacciones, evaluación, etc.) a un análisis reflexivo que identifique posibles sesgos de género (invisibilización de las mujeres y sus aportaciones, reproducción de estereotipos sexistas, naturalización de las relaciones de género, lenguajes no inclusivos, androcentrismo científico, etc.) y proponga alternativas (Mora y Pujal, 2014).

[...] aplicar la perspectiva de género a las ciencias conlleva analizar la construcción del conocimiento científico y revisar sus teorías y métodos desde una perspectiva crítica: cuáles son sus reglas, supuestos e hipótesis, en qué medida reflejan intereses de mujeres y de hombres, cuáles son sus consecuencias, para qué sirve un desarrollo científico, a quién perjudica o favorece... Lo que significa considerar los aspectos sociales como parte integral de la propia ciencia. (Bonilla, 2010, p. 66) 
La introducción de esta perspectiva permite, pues, romper dualismos y mitos como el de una ciencia social libre de emociones, y crea un espacio para la parcialidad, la autorreflexión y la diferencia (Martínez, 2003). La perspectiva de género como categoría analítica y explicativa en la investigación y la docencia es imprescindible para poder conseguir una ciencia mejor, más justa socialmente y menos coartada, así como para desarrollar una mejor praxis profesional con un compromiso ético y democrático más profundo.

Son diversas y múltiples las aportaciones que se han hecho desde epistemologías feministas, sobre todo a nivel de investigación, que dan lugar a una variedad de posiciones heterogéneas y diversas. Figuras importantes en los inicios de este repensar la ciencia han sido Harding (1987) o Haraway (1995). Todas ellas comparten una epistemología basada en la democracia y en la resistencia a los discursos dominantes (Colás, 2004).

El paradigma feminista se centra en la crítica a la ausencia de mujeres en el mundo de la ciencia, así como en la defensa de una ciencia y una epistemología feministas y en el análisis de sesgos androcéntricos en los métodos y las teorías científicas, incorporando una nueva forma de analizar la realidad en la que las relaciones de género constituyen un elemento fundamental (Colás, 2004). Una de las principales críticas ha sido y sigue siendo cuestionar la existencia de conocimientos generales y universales, generalizables, sobre nuestras realidades. Se considera que todos nuestros conocimientos son locales y temporales (Martínez, 2003). Como dice Lupton (citada en Denzin y Lincoln, 2000, p. 160-161): «la cuestión no consiste en buscar una cierta "verdad", sino en descubrir las variedades de verdad que operan, en poner de relieve la verdad como algo transitorio y político, y en enfatizar la posición de los sujetos como fragmentaria y contradictoria».

Este paradigma feminista propone como ejes principales:

- Reconocer las formas de identidad y de experiencia en la formación del conocimiento.

- Incorporar valores excluidos en la producción predominante de la ciencia.

- La necesidad de ver y conocer desde el punto de vista propio.

- La intervención en la producción de la ciencia y la tecnología, así como en la creación epistemológica.

- Las verdades situadas y el reconocimiento de la parcialidad y el posicionamiento en la producción del conocimiento.

Siguiendo a Mies (1983) y Harding (1987), para desarrollar una metodología feminista es necesario:

1. Reemplazar una investigación libre de valores, de neutralidad y de indiferencia hacia los «objetos» de investigación por una parcialidad consciente, que se logra por medio de una identificación parcial con los objetos de investigación.

2. Transformar la relación vertical entre investigador y «objetos de investigación» por una visión desde abajo, donde trabajar desde relaciones más equitativas y justas. 
3. Reemplazar el conocimiento de espectador contemplativo y no involucrado por una participación activa en las acciones, los movimientos y las luchas para la transformación social y de las relaciones de género.

4. Trabajar por un proceso de investigación concienciador que permita empoderar a las mujeres, problematizando situaciones, procesos y acciones de investigaciones que van en una dirección contraria.

5. Necesidad de incorporar las experiencias femeninas como recurso para el análisis social y también educativo, además de colectivizar estas experiencias superando el individualismo, la competitividad y el profesionalismo de los estudios actuales.

Hasta ahora, a nivel de docencia universitaria, la perspectiva hegemónica en el contexto catalán ha sido androcéntrica, primando la provisión, el objetivo de la cual es lograr lo establecido en el programa docente sin atender al conocimiento de las necesidades de la otra persona. En otros niveles educativos, se están introduciendo cambios que incorporan aportaciones de pedagogías críticas y pedagogías de género que están impactando en menor medida en la educación superior. Introducir una pedagogía de género en este ámbito implica dar un giro que permita introducir elementos ya señalados, así como trabajar la atención, el conocimiento y el cuidado en las aulas. El cuidado ha sido para el paradigma feminista un eje fundamental a trabajar. Por cuidado entendemos la relación social que va orientada a la atención y a la satisfacción de las necesidades de otro ser (Mora y Pujal, 2014). Incorporar la noción de atención y cuidado en la docencia conlleva tener en cuenta las necesidades de formación de las personas participantes, ver cómo se cumplen e ir modificando la acción formadora con este fin. Tener únicamente una óptica de provisión implicaría negar esas necesidades singulares de los individuos. En este caso, se apuesta por una combinación de ambas lógicas.

Según Klebesadel y Kempfert (Luxán y Biglia, 2011), a nivel metodológico, introducir una pedagogía de género implica:

- Estimular la autorreflexión para enfatizar la relación entre los contenidos curriculares y las propias vidas.

- Facilitar la colaboración para mejorar los conocimientos.

- Romper las barreras de los prejuicios.

- Comprometer a las clases con sus comunidades para provocar cambios sociales en ellas.

Se añadiría a lo anterior situar las relaciones de autoridad y poder en el centro del análisis. Esto conlleva plantearse las relaciones que se dan en el aula y cómo las transformamos para democratizar el conocimiento y desplazar la relación entre objeto (estudiante) y sujeto (docente) a una relación entre sujeto y sujeto. Hacer este ejercicio implica partir del reconocimiento mutuo como sujetos activos en la producción de conocimiento. Crear otra relación en el aula requiere de un aprendizaje que tiene que ver con sentirse parte de un 
colectivo, aprender a coconstruir el conocimiento y trabajar conjuntamente para ello, respetando todas las subjetividades (Luxán y Biglia, 2011).

\section{Objetivo}

El principal objetivo de este artículo es explorar el relevante papel que la introducción de metodologías y pedagogías feministas puede tener en el aumento de la calidad de la docencia. Para conseguir este objetivo, se analizaron los cambios y las aportaciones en cuatro ejes: la relación pedagógica, las metodologías usadas, los conocimientos impartidos y la participación.

La propuesta de innovación docente que se ha analizado se ha materializado en tres talleres participativos de 50 horas cada uno sobre perspectiva de género, con 25 participantes por taller. Se han hecho cuatro ediciones desde el curso 2012-2013 hasta el curso 2014-2015, que han sido organizadas por el Observatorio de la Igualdad y el Centro Joan Rivière en Psicología y Género, de la Universitat Autónoma de Barcelona. Estos talleres se ofertaron como actividades de reconocimiento académico para los grados.

Los temas que se impartieron en los talleres fueron: Psicologia y género, Violencia machista y Salud y género. En cada taller se contó con formadoras de grupos de investigación con perspectiva de género, personas de entidades especializadas en la materia de la propia universidad y profesionales en activo que incorporan dicha perspectiva en su práctica.

\section{Método}

El método utilizado para presentar la experiencia docente de los talleres impartidos se basa en dos narrativas colectivas de personas formadoras y participantes. Este diálogo entre diferentes miradas parciales permite acercarnos a la experiencia vivida desde diferentes posiciones y evaluarla de una forma más completa y profunda. Construir narrativas colectivas como método y proceso de investigación está relacionado con la epistemología feminista, que trata de romper la diferenciación entre sujeto y objeto y el realismo ontológico que postula la existencia de una realidad objetiva.

Las narrativas presentadas se han construido a partir del análisis de los datos recolectados durante la realización de cinco grupos de discusión, dos con formadoras y tres con personas participantes. Para los grupos de discusión se utilizó un guion de entrevista semiestructurado dividido en cuatro ejes: la relación pedagógica, las metodologías usadas, los conocimientos impartidos y la participación. Antes de cada grupo de discusión se explicaron los objetivos del estudio y se obtuvo un consentimiento informado de las participantes, donde se garantizaba la confidencialidad y el anonimato en la recogida y el análisis de la información.

Una vez realizadas y transcritas las entrevistas con los grupos, se hizo un análisis categorial de contenido semántico (Vázquez, 2000) con el soporte del programa Atlas Ti. Este tipo de análisis consiste en la transformación de los 
datos brutos en información útil a través de la fragmentación del texto y en el establecimiento de unidades de significado semántico por analogía. Es decir, se reducen los datos masivos obtenidos a denominadores comunes (códigos o categorías) vinculados jerárquicamente, lo que permite hacer un análisis descriptivo de los resultados en relación con el marco teórico y los objetivos de investigación (Penalva y Mateo, 2006). La finalidad de este análisis es obtener una vista condensada de los datos. El resultado es la construcción de una serie de temas preanalíticos en base a dos requisitos: a) la coherencia de las categorías temáticas con los objetivos de investigación y $b$ ) la generatividad (donde el sistema de clasificación proporciona una nueva visión de los datos). A partir de estas categorías se construyeron las narrativas que se consensuaron con los participantes y las participantes y que reflejan el análisis realizado.

La muestra fue de 20 personas (11 estudiantes y 9 formadoras). Con el objetivo de garantizar la máxima heterogeneidad y variabilidad, para seleccionar la muestra de estudiantes se utilizaron diferentes criterios: sexo, año académico, estudios y participación en los talleres.

Para el grupo de formadoras, y con la misma voluntad, se utilizaron los siguientes criterios: ámbito de conocimiento, institución en la que trabajan, profesión, experiencia como formadoras y taller en el que participaron.

\section{Resultados}

A continuación se presentan las dos narrativas construidas a partir de los resultados de los grupos de discusión sobre las aportaciones de las pedagogías y de las metodologías feministas a la calidad de la docencia.

\subsection{Primera narrativa: Experiencia y procesos del estudiantado}

Esta primera narrativa recoge los cuatro procesos principales de las personas estudiantes: la construcción colectiva de conocimiento, la construcción de una relación pedagógica desde la igualdad, la incorporación de la perspectiva de género como transversal y el proceso de devenir agentes activos de cambio.

\section{Construyendo colectivamente el conocimiento: Empoderamiento del estudiantado}

En la conversación sobre la experiencia vivida se narra cómo, a lo largo de los talleres, se va transformando la participación de las diferentes personas del grupo. Se da un proceso de cambio en la posición subjetiva hacia una posición activa en el aprendizaje, transformándose la forma de estar en el aula y encontrando su propio espacio para intervenir, y hacerlo no solo para plantear dudas, sino también para generar debate, contraargumentar una opinión, abrir temas, exponer experiencias, etc. El aula se convierte en un diálogo entre diferentes saberes, posiciones, conocimientos, etc. Cada una discute a partir de su conocimiento situado, su experiencia y su bagaje, conectando diferentes saberes, identificándose con la otra persona, analizando las experiencias propias como 
colectivas y empoderándose como sujetos a partir de la construcción conjunta de conocimiento. Este cambio de posición también genera una reflexión crítica sobre la propia posición en otros contextos de aula.

A mí una cosa que me gustó mucho era que todo lo que aprendíamos era por nosotros mismos, quiero decir, que tú [refiriéndose a la formadora] en ningún momento nos ibas a adoctrinar, «no, esto es así y ya», sino que, con las preguntas, lo íbamos sacando. Esto nos ayudaba a que nos quedara más, a que lo viéramos todo más aplicado a la vida real, y, lo que dice ella del aprendizaje, estamos acostumbrados a un tipo de modelo educativo en el que "esto es así, porque lo dicen los libros». Yo lo he vivido siempre igual y creo que es una de las maneras que debe cambiar mucho, esta forma de enseñar. Yo creo que era un método muy diferente, y que los estudiantes aprendemos más que si te lo están diciendo. $(\mathrm{P} 4, \mathrm{GD} 1)^{1}$

Esta nueva forma de estar en el aula hace emerger un proceso de valorización y conciencia de los propios saberes, así como de ampliación de los mismos. Se profundiza en los conceptos trabajados, cosa que permite entrelazar temas, preocupaciones y necesidades y que emerjan nuevas dudas y debates que se van integrando en el conocimiento del grupo.

\section{Construyendo una relación pedagógica desde la igualdad:}

\section{Posiciones subjetivas y relaciones de poder}

Se narra inicialmente una idea del poder y de la autoridad como relación jerárquica entre formadoras y estudiantes. Frente a ese poder y a esa autoridad se da la obediencia y la escucha, con una distancia enorme entre estas dos posiciones. En los talleres esta visión se subvierte, de modo que sus participantes devienen conscientes de cómo el poder se construye y se reconstruye en nuestras relaciones.

[...] cuando se habla de poder, la gente piensa inmediatamente en una estructura política, en un gobierno, en una clase social dominante, en el señor frente al esclavo, etc. Pero no es en absoluto en esto en lo que yo pienso cuando hablo de relaciones de poder. Me refiero a que, en las relaciones humanas, sean cuales sean -ya se trate de una comunicación verbal, como la que estamos teniendo ahora, o de relaciones amorosas, institucionales o económicas-, el poder está siempre presente: me refiero a cualquier tipo de relación en la que uno intenta dirigir la conducta del otro. (Foucault, 1984, p. 269)

En este sentido, el poder reprime, pero también produce, ejerce efectos sobre la gente, la subjetiviza. La posición de la persona formadora se iguala a las demás posiciones en un proceso donde todas las voces adquieren autoridad, aunque, por su experiencia y recorrido, pueda aportar cuestiones cualitativamente diferentes. Es más una guía que acompaña a los demás que una fuente

1. La P señala la persona del grupo que hizo la aportación y el GD indica el código del grupo de discusión. 
de saber en sí misma en un proceso de aprendizaje que tiene en cuenta las necesidades, los sentires, las experiencias y los ritmos diversos del aula.

La figura que tenemos en general del profesor o de la profesora es que es quien tiene el poder y parece que no podamos aportar mucho, porque "qué tengo que decir yo, si él sabe más». También hablamos bastante del poder, sobre qué influencia ejercías tú sobre nosotros y nosotras tratándolo con naturalidad, que realmente es así, que igual que tú un día dices una cosa que puede que no nos cuestionemos y que nos podríamos cuestionar o que sí que nos cuestionamos. Pero creo que lo quisiste tratar de igual a igual, de igualarte a nosotros y yo normalmente no tengo esta sensación, de la profesora como alguien próximo, sino que ella está allá y yo aquí, ella tiene el conocimiento y yo aprendo. (P3, GD1)

Esta transformación en las relaciones se hizo posible por el trabajo desde la atención, el cuidado, el respeto a las diferentes subjetividades, el no juzgar y la escucha activa. Se narra la sensación que las aportaciones y demandas eran recogidas y había espacio para ellas. Este espacio también se transformaba físicamente de manera continua para romper con la fijación de posiciones e ideas a través de las dinámicas de grupo. Esta forma de proseguir se confronta con la educación formal, donde perciben que no hay tiempo ni está permitido salir del tema.

Era como una burbuja, entrabas allí y explicabas cosas que eran realmente íntimas, o había gente que decía «esto no lo diré», y no importaba, no te estaban obligando. Pero sí que decías cosas que a mitad de la frase decías: « $\mathrm{U} U y$, uy, uy! ¿Qué estoy diciendo?», y luego salías de allí y tampoco nos hemos relacionado mucho más, pero estabas allí y compartías aquello. (P3, GD1)

El aprendizaje no se limitaba al aula y el aprendizaje no se limitaba al tema, porque si te dicen que este tema no toca, ya te están limitando. (P1, GD1)

Y yo creo que fue todo cuidado, con mucho tacto. Además, había como un silencio, no sé, como una intimidad... (P1, GD4)

A mí me llamo mucho la atención su lenguaje corporal [refiriéndose a la formadora]: la sonrisa, la postura física, el ocupar los espacios, la velocidad a la que se movía. Yo me fijaba y decía: « $U_{\mathrm{U}}$ !». Todo lo que hacía invitaba a ese cuidado ¿no? Yo me imaginaba, digo: «A lo mejor igual tiene facilidad para esto, pero lo ha tenido que trabajar mucho esto». (P4, GD4)

\section{Incorporando la perspectiva de género de forma transversal en el proceso} de aprendizaje pasado, presente y futuro

El estudiantado entiende la perspectiva de género como unas gafas nuevas que le permiten visibilizar y analizar las desigualdades de género que antes normalizaba, así como entender sus orígenes y sus consecuencias para poder transformarlas. Todo ello se convierte en una perspectiva transversal que incorporan en sus aprendizajes y en la vida cotidiana. 
Porque, claro, yo ya será mi punto de vista de todo lo que hago en mi vida. De todas mis relaciones sociales y de mi yo estudiante. Todos mis trabajos tendrán esta perspectiva. Cuando veo una película, ya le encuentro esta perspectiva, cuando leo un libro, también. (P2, GD3)

Esa mirada, esa perspectiva y ese conocimiento de género dan lugar a un proceso de revisión de los conceptos adquiridos hasta entonces desde una visión crítica, cuestionándose qué es ciencia, quién la produce, qué incluye y qué excluye, desde dónde se produce y con qué fines. Es decir, tiene lugar una crítica epistemológica a la supuesta neutralidad, objetividad e imparcialidad de los saberes recibidos.

Frente a este escenario, las estudiantes se plantean, por un lado, la necesidad de repensar la propia formación a nivel de contenidos, metodologías, etc., y, por otro, la necesidad de introducir la perspectiva de género en la formación de las profesionales. Empiezan a pensar que ignorar las relaciones de género puede llevar a una mala praxis, ya que es muy difícil abordar ciertos problemas sin tenerlas en cuenta. Esta reflexión genera, en algunos casos, la búsqueda de espacios formativos reglados o no reglados sobre esta perspectiva dentro de su disciplina.

Es que es un gran tema, es qué se espera de ti, son las exigencias sociales. Por tanto, casi todo girará alrededor tuyo, no puedes hablar solo de "¿̇y en tu casa cómo eran las cosas?», porque tus padres también forman parte de una sociedad y después la sociedad también les está diciendo cosas, y parte del problema tiene este origen. Si no tenemos esta perspectiva como psicólogos, estamos tratando a ciegas, ¿¿no? (P3, GD1)

\section{De la formación a la acción: Deviniendo agentes activos en la transformación de las relaciones de género}

Se narra que los conocimientos generados en los talleres traspasan el aula a través de diferentes procesos: la identificación, la autorreflexividad y la transformación. Se exponen situaciones en las que empiezan a adoptar una mirada crítica frente a ciertos fenómenos cotidianos relacionados con la reproducción de las normas de género e intervienen sobre ellos en diferentes contextos — con la familia, como monitores de niños y niñas, con la pareja, con los amigos y las amigas, etc.- , generando discursos y prácticas transformadoras. Dejan de considerar permisibles, normales o naturales ciertas conductas e intentan subvertirlas volviéndose agentes activos de cambio. Un proceso que no está exento de contradicciones, impotencias, frustraciones, malestares, etc.

Yo creo que es un poco como que te cae la venda y una vez te das cuenta de estas cosas..., pues igual antes en una conversación no te darías ni cuenta, y ahora, aunque no quieras, suena una alarma y dices: «iDios mío! ¡iQué están diciendo!?». (P5, GD4)

Realmente vives en esto y lo llevas como puedes. Con los amigos y las amigas, entras en el debate: «¿y esto por qué?», y vas haciendo las preguntas. A veces va bien y otras no, y lo ves, y genera una impotencia, si ni tan solo se ha 
planteado porque piensa como piensa... ¿Quién soy yo para ir a decirle cómo tiene que hacer las cosas? (P4, GD1)

Es más fácil hablar de neurociencia, de tecnologías, porque todo el mundo lo acepta. «;Cuántos conocimientos tienes!», te dicen. Tienes prestigio. No obstante, hablas de eso, has hecho una reflexión brutal, has pensado mucho, lo has trabajado y la gente te dice: «Dedica tu tiempo a una cosa más productiva». (P1, GD1)

Y también noté la función de... A parte de conocer la problemática, verte tú en el espejo, sobre todo en las sesiones de teatro. Esto lo noté. Porque estábamos usando la autorreflexividad todo el rato. (P4, GD4)

En este sentido, emerge la necesidad de actuar, de transformar las relaciones de poder de género hacia relaciones más equitativas y justas. Es un aprendizaje para la acción, puesto que, en tanto que nuestras prácticas en las relaciones de género son inestables, dinámicas y cambiantes (Butler, 2007), se pueden transformar, y así lo expresan las participantes.

Es de las pocas cosas que te llevas más allá de clase, porque normalmente te quedas aquí. Cuando vas a estudiar, abres el libro y estudias, pero más allá no te sirve. A mí me ha servido a nivel personal, como monitora de niños y niñas me ha ido muy bien, porque detrás de la simple dinámica de formar grupos para un juego, de hacer uno de niños y otro de niñas, que sabes que ganarán los niños, hay implícitas muchas cosas, aunque tengan cinco años. Y, luego, que haya una persona, que hay más de una, que diga: «Al loro, que no queremos promover esto, pero lo estamos poniendo aquí». (P2, GD1)

\subsection{Segunda narrativa: Experiencia y procesos de las formadoras}

Esta segunda narrativa recoge los tres procesos principales que se narran en la conversación entre las personas formadoras: la interrogación sobre el propio saber desde una perspectiva de género, el habitar entre lo formal y lo informal y el impacto en subjetividades diversas de sus acciones.

\section{Interrogándose sobre el saber desde la perspectiva de género:} Apertura a metodologías participativas

Las formadoras, como productoras y transmisoras de conocimiento, reflexionan sobre qué significa la perspectiva de género para ellas, cómo la aplican, para qué les sirve y qué aporta esta perspectiva al saber.

Yo creo que la perspectiva de género lo que hace es cuestionar lo cotidiano, cuestiona lo que se supone que debe ser, y abre, ensancha, tramas narrativas para preguntarse quién es uno mismo. Una pregunta que, aunque uno no se la hace, se la hacen. Yo creo que abre espacios de hábito en cosas nuevas, replantearse incluso hasta las propias reacciones que uno tiene frente a cosas o temas diversos: la paternidad, la maternidad, los vínculos, los propios derechos, lo que debe ser como mi rol, o no. Yo creo que de alguna manera da esa 
plasticidad o ese proceso de no identificarse continuamente con lo que se debe ser. Y yo creo que eso le pasa a todo ser humano, sobre todo porque ya nos van posicionando siempre. Es una dialéctica entre dónde nos ponen y dónde nos queremos poner nosotros. Y yo creo que te hace pensar, por lo menos, dónde me estás poniendo, o sentirse, ¿no?, porque cada vez estamos más desconectados de lo que sentimos, de cómo estamos, del cuerpo. (F4, GD5) ${ }^{2}$

En este proceso de interrogación sobre la perspectiva de género y el saber se ponen en juego posturas epistemológicas en torno al conocimiento situado (Haraway, 1995), se reflexiona sobre las dicotomías entre público y privado, reflexión y acción, sobre la interrogación crítica al conocimiento, el androcentrismo científico y la transversalidad del género.

Una de las cuestiones que se narran es qué implica partir de una perspectiva de género también a nivel metodológico, ya que, tal como apunta una de las formadoras: «El conocimiento y las metodologías en la perspectiva de género van muy unidos» (F1, GD2).

El aspecto metodológico influye, porque a veces se reproducen los mismos patrones que en el ámbito formal. Puedes dar una perspectiva crítica sin una metodología crítica. (F4, GD2)

En este sentido, hablan de las potencialidades que tiene partir de la propia experiencia de las participantes, de sus prácticas, y no de discursos teóricos aprendidos.

Yo creo que la herramienta más potente que tenemos a nivel metodológico es de alguna manera incorporar una parte de la experiencia. Si haces un análisis de caso sobre papel, para mí tiene más potencia analizar un caso que está pasando en un grupo en ese momento. [ ...] Cuando trabajas género, en todas las aulas hay temas de género. Entonces, es bueno, ponemos la carne en el asador y trabajamos desde cómo estamos viviendo esto. ¿Qué pasa? ¿Por qué hay una valoración tan positiva de los talleres cuando lo hacemos? Porque es desde la experiencia que aprendes. [...] Trabajar en la teoría es muy sencillo y ver cómo los discursos políticamente correctos los tiene todo el mundo es clarísimo, pero cuando vamos a la práctica somos totalmente incompetentes. Que las relaciones deben ser igualitarias, todo el mundo lo sabe, ahora, ponte aquí y pon un límite, y a ver cómo lo haces. (F3, GD5)

Esta mirada analítica desde el género que se desarrolla a lo largo de los talleres y que parte de la experiencia empieza a problematizar el mismo conocimiento y el modo como se construye (Biglia, 2005).

Para mí, pasa por cuestionarse: ¿qué es el conocimiento? ¿Qué entra y qué no entra? ¿Qué cosas se valoran y cuáles no? ¿Cómo se ha conseguido el conocimiento? ¿Con qué finalidades? [...] ¿Qué relaciones de poder se mueven en las universidades? ¿Dónde han quedado los saberes femeninos? (F5, GD2)

2. La F señala la formadora del grupo que hizo la aportación y el GD indica el código del grupo de discusión. 
En este sentido, se considera necesario que la perspectiva de género atraviese todos los ámbitos del conocimiento, de la investigación y de la formación. Se insiste en que no quede relegada a unos temas o a unas áreas de saber o a la voluntad o a la formación de algunas profesionales, sin negar las dificultades ni las resistencias que esto supone, igual que han señalado otros estudios (Verge, Ferrer-Fons y González, 2017).

Es una visión transversal, pero es complicado, porque si pones asignaturas individuales de género vuelves a fragmentar y a pedir transversalidad cuando no hay la formación necesaria... (F5, GD2)

Y yo creo que esa es la gran incógnita, por lo menos para mí, cómo se puede desarrollar esto transversalmente, y por eso el hacer los cursos que hacemos acá, ¿̇no? Qué público tan diverso... y si tendría un carácter sensibilizador, pero yo creo que tiene también un carácter de movilizar y de prestarle atención, porque yo creo que es un tema que cruza todas las áreas, ¿̨no? (F4, GD5)

Yo creo que, en el género como en el poder, lo más importante para mí a la hora de hacerlo transversal es poder hacer visible lo invisible. (F3, GD5)

Finalmente resulta presente la relación entre el conocimiento y la transformación social (Biglia, 2005) en el campo de la investigación activista feminista, y se lleva al terreno de la formación, donde el proceso de transformación es descrito como bidireccional.

El modo como yo me coloco delante del mundo, ¿no? Hay un tema de compromiso. (F1, GD2)

De compromiso político y de transformación social en el propio conocimiento. (F5, GD2)

Y poder hablar de nosotras como sujetos que también nos transformamos, [...] que podamos decidir a través de la educación quién queremos ser. (F1, GD2)

\section{Habitando el ámbito formal e informal:}

Gestión de espacios, negociación e identidades en tránsito

En los diálogos entre formadoras se detecta una conceptualización de dos espacios diferenciados y confrontados: el ámbito formal -la docencia reglada dentro de los grados - y el informal — las actividades de reconocimiento académico, como los talleres propuestos que tienen un marco más flexible y abierto en cuanto a metodologías, estructura y contenido- . En la narrativa se pone de relieve y se cuestiona el papel del sistema educativo y las restricciones y los límites que encuentran como docentes en su práctica formativa.

El problema del ámbito formal es que alguien ha decidido cómo tienen que ser las cosas, es interesante quién decide. [...] Esto te obliga a una transformación del conocimiento y del modo en que lo transmites. Yo que siempre he sido de 
carácter práctico, ahora tengo que enseñar sin poder hacer, y esto es contrario a mi planteamiento. (F2, GD2)

Yo creo que el sistema educativo no es neutro, ni objetivo, ni espera dar el mejor aprendizaje, sino que está hecho para finalidades [...], la transmisión de unos valores determinados. (F5, GD2)

Los alumnos ya no esperan que haya oportunidades en el campus para aprender de otra forma. (F1, GD2)

El contexto informal se conceptualiza como un espacio más libre de las limitaciones estructurales de la docencia formal, una alternativa que se plantea como transformadora en una doble dirección: de la propia persona y del mismo sistema de docencia. Se expresa el modo en que las formadoras gestionan la intervención en ambos espacios, reconociendo los sentimientos, las actitudes y las sensaciones diferenciadas en unos y otros y su impacto. Se destaca la posibilidad de construir un "yo docente» más libre y de replantearse la propia posición en el ámbito formal.

La informalidad a mí me ha abierto puertas, para repensar el propio currículo y lo que hago en las clases. (F5, GD2)

El espacio informal, por favor que siga siéndolo, porque posibilita cosas que el espacio formal no nos está posibilitando. (F1, GD2)

Yo creo que sí que son una alternativa en el momento actual, pero pienso que ya está bien de trabajar estas temáticas desde el ámbito no formal. Nosotras estamos en la universidad, estamos cerca, y realmente hay caminos. Vamos a empezar a hacer incidencia para transformar el modelo educativo de una vez. (F3, GD2)

O sea, ¿transformar la universidad desde estos espacios? (F1, GD2)

Aunque se perciben como espacios más libres para poder repensar cómo habitar el aula, cómo construir relaciones más equitativas en su seno, cómo transformar las maneras de enseñar y aprender, etc., los talleres no dejan de inscribirse como actividades de reconocimiento académico en un marco institucional y formal que tiene estructuras, normas y reglas de evaluación. Las formadoras reconocen y expresan estos límites generando una doble censura: la propia - al tener interiorizados una serie de elementos del ámbito formal- y la de la institución —que se impone con la evaluación.

Al final piensas que podrías haber hecho mucho más, porque a lo mejor te estabas autocensurando. Tú misma te regulas con lo que parece que el contexto espera de ti. (F1, GD2)

Hay una parte de dificultad, porque estás caminando en contra de todo. Entonces, en el momento en que, en mi caso, por ejemplo, en el momento en que cambias el enfoque, dejar un espacio para poder expresar las emociones, en un contexto académico, es muy difícil. Si estoy haciendo un taller, que no tengo 
que evaluar, hacer una rueda de cierre y pedir que cada uno diga una palabra acerca de cómo se ha sentido o de lo que le ha aportado el taller, me es muy fácil, pero cuando estoy dando clase, donde los estudiantes deben ser evaluados... ¡Uf? [...] Si aplico verdaderamente la perspectiva de género de forma transversal, debo abrir espacios para que cada uno exprese lo que siente con lo que está haciendo en la asignatura, pero, ¿cómo lo evalúo? Todo el sistema está pensado, aunque incluyamos una perspectiva de género, desde unas premisas que son la objetividad, el rendimiento, la productividad, etc. (F3, GD5)

Reflexionando criticamente sobre los múltiples impactos en subjetividades diversas: formadoras, estudiantado e institución

Las formadoras en los grupos de discusión hablan de tres impactos que ha generado la experiencia de los talleres:

1. Hacia ellas mismas, en el sentido de repensar su "yo formadora», las pedagogías usadas, la gestión del poder en el aula, etc.

2. Hacia las estudiantes, que tiene que ver con el impacto generado en la otra persona.

3. Hacia la institución, en términos de qué cambios genera abrir espacios de este tipo y hablar de género en una institución universitaria.

En este sentido, destaca su percepción de estar movilizando y generando reflexión en la otra persona, sus temores alrededor de este impacto y la responsabilidad que conlleva, así como la necesidad de asegurar la reflexión crítica de las estudiantes.

Yo tengo la sensación de que moviliza, no a todas, jeh!, porque es imposible que les toque a todas, pero que moviliza cosas seguro. Y quizás es solo una persona, pero al ser una persona que hace un cambio en un contexto grupal, ya hay un punto en que toca el grupo. [...] Tengo esa sensación, porque cuando no pasa, creo que se nota y me voy frustrada. (F3, GD5)

Este impacto no está exento de contradicciones y miedos, lo que provoca una revisión crítica de la misma práctica de las formadoras. Se discute sobre las consecuencias en la otra persona y si realmente se están dando las herramientas necesarias que ayudan a crear determinadas miradas críticas para la reflexión o si se están generando nuevos adoctrinamientos.

Pero esto da miedo. Cuando alguien sale de un taller y dice que le has cambiado la vida, piensas: «A ver qué sale de aquí». (F5, GD2)

Sí, es una responsabilidad muy grande. (F1, GD2)

Además, son poco críticos contigo, con lo que yo digo. (F5, GD2)

Es muy complaciente... Nadie te cuestiona, nadie se atreve a contestarte, cuando precisamente lo que quieres es que sea contestado, discutido y reflexionado. (F1, GD2) 
Alguna vez sí que tuve la sensación de que lo que estábamos haciendo era un adoctrinamiento a la inversa. (F3, GD2)

Esta reflexión implica también un proceso de cuestionamiento y concienciación sobre la propia posición de formadora en el aula: ¿cómo se gestiona el poder y la autoridad que una tiene en ese espacio?, ¿cómo emerge y negocio ese ejercicio con las otras personas?, ¿es posible una relación igualitaria en el aula?, ¿reciben la misma atención todas las voces del aula?, etc. Las formadoras hablan de estrategias y de herramientas para aprender a gestionar y visibilizar todas estas cuestiones en el marco del aula incluyéndolas en el contenido como parte del aprendizaje.

Pero no tienes una posición neutra. Muchas veces tu voz no tiene el mismo poder que las otras. (F4, GD2)

Pero puedes decirlo, no esconderlo, que es lo que hace la pedagogía tradicional, y multiplicar las otras voces. (F1, GD2)

Por último, y en relación con el contexto donde esta educación se está dando, las formadoras se preguntan sobre el impacto que ejerce abrir estos espacios para poder normalizar el hecho de hablar sobre el género (contenido) y desde el género (metodología) en la universidad. Al tratarse de ámbitos que generan ruido por estar fuera de la norma como espacio docente, este ruido impacta en ellas mismas, en las estudiantes y en la propia institución, ya que alumnas y formadoras, como hemos visto en las narrativas, empiezan a problematizar el funcionamiento de la propia institución universitaria.

Luego hay lo que tiene que ver con empezar a hacer presentes estos temas dentro de lo que es la agenda académica, y más todavía conectarlo con la psicología. O sea, yo en la carrera nunca vi género. No existía. Bueno, existía, pero nadie lo tocaba. Entonces yo creo que sí, es una tarea importante, y eso también tiene un carácter simbólico, de poner esa pieza y de que empiece a estar presente y que se empiece a volver normal cuestionarse sobre estas cosas. (F4, GD5)

\section{Discusión y conclusiones}

El presente estudio muestra que los resultados no pueden separarse de los procesos, ya que estos deben ser también objeto de atención y de análisis. La persona enseñante debe tratar de observar y entender aquello que el alumnado conoce y cómo actúa, y no limitarse a considerar únicamente sus carencias, su falta de conocimiento. Solo siendo capaz de articular lo que se sabe con lo nuevo que se aprende se puede conseguir un proceso de transformación individual. Del mismo modo, la persona enseñante, al ejercitar este diálogo y verse involucrada en este proceso transformador, puede ella misma reconsiderar sus conocimientos y su forma de construirlos y reconstruirlos.

El presente trabajo nos permite repensar la calidad de la formación superior desde perspectivas más centradas en las personas, en su potencial de desarrollo 
y en la generación de nuevos conocimientos y perspectivas, más que en su reproducción.

La principal conclusión de este estudio es que la introducción de pedagogías, epistemologías y metodologías feministas en la docencia permite transformar el conocimiento anterior, la propia mirada analítica hacia el entorno y las propias acciones en él, igual que señalan otros estudios similares (DonosoVázquez y Velasco Martínez, 2013; Colás, 2004). Aplicar la perspectiva de género, tanto en el contenido como a nivel metodológico, abre posibilidades para reflexionar de forma crítica desde la propia experiencia y posición sobre las relaciones de género del contexto; permite repensar las propias prácticas para devenir sujetos activos de cambio y transformar las relaciones de género; genera un impacto en el aprendizaje a nivel subjetivo, profesional e institucional, y transforma las relaciones pedagógicas en el aula, corresponsabilizándose del aprendizaje y coconstruyendo el conocimiento.

De esta manera, el saber generado y las metodologías utilizadas aparecen como una alternativa. $\mathrm{Al}$ aplicar esta perspectiva en los talleres, estos emergen como un espacio de transformación individual y colectiva, de lo personal y lo social, de lo doméstico y lo académico; un espacio para transformar las relaciones pedagógicas potenciando su democratización y profundizando en el conocimiento. Se hace posible el trabajo desde la atención, el cuidado, desde el diálogo con la otra persona en un proceso constructivo, donde tienen cabida todas las inquietudes y necesidades, y donde el conocimiento es construido colectivamente, en la misma dirección que señalan otros estudios mencionados en el presente artículo (Mora y Pujal, 2014). Se transgrede la división entre teoría y práctica, a la vez que se transgreden y se transforman las relaciones de género en la práctica profesional, académica y personal. El aprendizaje va más allá del aula, se mentaliza y se corporativiza, y también se explicita cómo somos sujetos de género y cómo, a través de las propias prácticas, reproducimos o transformamos estas relaciones.

Por último, cabe mencionar que el campo de estudio en el que se ha centrado la investigación es un ámbito donde no hay mucha literatura que plantee qué implica repensar la docencia universitaria a nivel metodológico y pedagógico, y menos aún con perspectiva de género, como sí pasa en otras etapas de aprendizaje. Por lo tanto, sigue siendo una asignatura pendiente para pedagogos y pedagogas, docentes con formación en perspectiva de género y todas las personas con compromiso con la igualdad y la diversidad.

\section{Referencias bibliográficas}

Biglia, B. (2005). Narrativas de mujeres sobre las relaciones de género en los movimientos sociales. Tesis doctoral. Barcelona: Facultad de Psicología. Universidad de Barcelona.

Bonilla Campos, A. (2010). Psicología, diferencias y desigualdades: Límites y posibilidades de la perspectiva de género feminista. Cuadernos de Psicología, 12(2), 65-80.

<http://dx.doi.org/10.5565/rev/qpsicologia.806> 
Butler, J. (2007). El género en disputa: El feminismo y la subversión de la identidad. Madrid: Paidós, 1990.

Colás Bravo, P. (2003). Investigación educativa y crítica feminista. Agora Digital, 6, 11-12.

- (2004). La construcción de una pedagogía de género para la igualdad. En M.A. Rebollo e I. Mercado. Mujeres y desarrollo en el siglo Xxi: Voces para la igualdad (pp. 275-292). Madrid: MacGrawHill.

Denzin, N. y Lincoln, Y. (2000). Handbook of qualitative research. Thousand Oaks, CA: Sage.

Donoso-Vázquez, T. y Velasco-Martínez, A. (2013). ¿Por qué una propuesta de formación en perspectiva de género en el ámbito universitario? Profesorado: Revista de Currículum y Formación del Profesorado, 15(1), 71-88.

Foucault, M. (1984). La ética del cuidado de uno mismo como práctica de la libertad. Concordia, 6, 99-116.

Grünberg, L. (2011). From gender Studies to gender IN studies: Case Studies on GenderInclusive Curriculum in Higher Education. Bucarest: UNESCO - CEPES Studies on Higher Education.

Guil Bozal, A. (2016). Género y construcción científica del conocimiento. Historia de la Educación Latinoamericana, 18(27), 263-288. <https://doi.org/10.19053/01227238.5532>

Haraway, D. (1995). Ciencia, cyborg y mujeres: La reinvención de la naturaleza. Madrid: Cátedra.

Harding, S. (1987). Feminism and methodology: Social science issues. Bloomington: Indiana University Press.

LuxÁN, M. y Biglia, B. (2011). Pedagogía ciberfeminista: Entre utopía y realidades. Teoría de la Educación y Cultura en la Sociedad de la Información, 12(2), 149-183.

Martínez, M. (2003). Epistemología feminista y postmodernidad. Cinta Moebio, 16, 50-56.

Mies, M. (1983). Towards a methodology for feminist research. En G. Bowless y K. Renate. Theories of Women's Studies (pp. 117-139). Londres: Sage Publications.

Mora, E. y Pujal, M. (2014). El cuidado y la provisión en el proyecto docente universitario. Un ejemplo: la asignatura. Las clases sociales y la estratificación del grado de Sociología. En III Jornada de Innovación en Género: Docencia e Investigación (pp. 105-126). Vigo: Unidad de Igualdad. Universidad de Vigo.

Penalva, C. y Mateo, M.A. (2006). Tècniques qualitatives d'investigació. Alacant: Universitat d'Alacant.

RuÉ, J. (2015). Entornos de aprendizaje digitales y calidad de la educación superior. Barcelona: UOC.

VÁzQuez, F. (2000). El análisis de contenido temático: Objetivos y medios en la investigación psicosocial. Barcelona: Universitat Autònoma de Barcelona.

Verge, T.; Ferrer-Fons, M. y GonzÁlez, M.J. (2017). Resistances to Mainstreaming Gender into the Higher Education Curriculum. European Journal of Women's Studies, 9 (enero).

<https://doi.org/10.1177/1350506816688237> 